\title{
Using Cherenkov imaging to monitor the match line between photon and electron radiation therapy fields on biological tissue phantoms
}

\author{
Yi Li $\odot,{ }^{\text {ab, }, c}$ Hongjun Liu,, a,d,* Nan Huang, ${ }^{\text {a }}$ Zhaolu Wang, ${ }^{a}$ \\ and Chunmin Zhang ${ }^{\mathbf{b}, *}$ \\ ${ }^{a}$ Chinese Academy of Sciences, Xi'an Institute of Optics and Precision Mechanics, \\ State Key Laboratory of Transient Optics and Photonics, Xi'an, China \\ ${ }^{b} X i$ ' an Jiaotong University, School of Physics, Xi'an, China \\ 'University of Chinese Academy of Sciences, Beijing, China \\ ${ }^{\mathrm{d} S h a n x i}$ University, Collaborative Innovation Center of Extreme Optics, Taiyuan, China
}

\begin{abstract}
Significance: Due to patients' respiratory movement or involuntary body movements during breast cancer radiotherapy, the mismatched adjacent fields in surface exposure regions could result in insufficient dosage or overdose in these regions, which would lead to tissue injury, excessive skin burns, and potential death. Cherenkov luminescence imaging (CLI) could be used to effectively detect the matching information of adjacent radiation fields without extra radiation or invasive imaging.

Aim: Our objective was to provide a biological experimental basis for monitoring matching of adjacent radiation fields between photon and electron fields due to introduced shifts during radiotherapy by CLI technique.

Approach: A medical accelerator was used to generate photon and electron fields. An industrial camera system was adopted to image the excited CLI signal during irradiation of chicken tissue with yellow (group A and group C experiments) or black color (group B experiment). The following introduced shifts were tested: $10,5,2$, and $0 \mathrm{~mm}$ toward superior or inferior direction. A model was introduced to deal with matching error analysis of adjacent radiation fields due to introduced shifts with adapted plans used to treat neoplasms of the right breast with supraclavicular nodes or internal mammary lymph node.
\end{abstract}

Results: The matching values between photon and electron fields were consistent with the tested introduced shifts during yellow chicken irradiation. In group A, average discrepancies were $0.59 \pm 0.35 \mathrm{~mm}$ and $0.68 \pm 0.37 \mathrm{~mm}$ for photon fields and electron fields in anterior/posterior (AP) direction, with $87 \%$ and $75 \%$ of measurement within $1 \mathrm{~mm}$, respectively. In group C, average discrepancies were $0.80 \pm 0.65 \mathrm{~mm}$ and $1.07 \pm 0.57 \mathrm{~mm}$ for oblique photon field with gantry angles of $330 \mathrm{deg}$ and $150 \mathrm{deg}$, with $66 \%$ and $65 \%$ of measurement within $1 \mathrm{~mm}$, respectively. The average discrepancies were $0.44 \pm 0.30 \mathrm{~mm}$ for electron field in the AP direction, with $94 \%$ of measurement within $1 \mathrm{~mm}$. The matching error introduced by the proposed method was less than $1.5 \mathrm{~mm}$ for AP fields and $2 \mathrm{~mm}$ for oblique incidence fields. However, the field matching could not be monitored with black chicken tissue irradiation due to a weak CLI signal that could hardly be extracted from background noise in group B.

Conclusions: CLI is demonstrated for the quantitative monitoring of the field match line on light biological tissue phantoms and has potential for monitoring of field matching in surface tissue during breast cancer radiotherapy.

(C) The Authors. Published by SPIE under a Creative Commons Attribution 4.0 Unported License. Distribution or reproduction of this work in whole or in part requires full attribution of the original publication, including its DOI. [DOI: 10.1117/1.JBO.25.12.125001]

*Address all correspondence to Hongjun Liu, liuhongjun@opt.ac.cn; Chunmin Zhang, zcm@xjtu.edu.cn 
Keywords: Cherenkov luminescence imaging; radiotherapy; field matching; biological tissue; charge-coupled device.

Paper 200268RR received Aug. 21, 2020; accepted for publication Nov. 16, 2020; published online Dec. 9, 2020.

\section{Introduction}

Recently, the incidence of breast cancer is increasing year by year. Radiotherapy can significantly reduce the postoperative recurrence rate of breast cancer. ${ }^{1}$ The radiation treatment region for advanced breast cancer includes breast or chest wall combined with regional lymph nodes. Irradiation of internal mammary lymph node or supraclavicular fossa (SCF) lymph nodes is applied to improve local control and to reduce the incidence of symptomatic disease in this region. ${ }^{2}$ In a radiotherapy plan, photon fields irradiated for the breast and internal mammary lymph node significantly increased the irradiated volume of the affected lung, even with volume-modulated arc therapy technique, which could reduce the dose to critical structures compared to traditional techniques while maintaining conformal and homogenous doses with a target volume within a few minutes of treatment. Therefore, photon fields and single electron field have often been irradiated for breast and internal mammary lymph nodes, respectively, ${ }^{3,4}$ which could significantly decrease the irradiated volume of affected lung during advance breast cancer radiotherapy in our department. These adjacent fields need matching under complex geometrical regions. However, due to patients' respiratory movements or involuntary body movements and irregular neck and chest contours, in addition to soft and shifty breast tissue during breast cancer radiotherapy, the mismatched adjacent fields in surface exposure regions could result in insufficient dosage or overdose in these regions, which may lead to tissue injury, excessive skin burns, and potential death. ${ }^{5,6}$

Nowadays, image-guided radiation therapy (IGRT) technique is developing rapidly, aiming to decrease the deviation of irradiation position caused by tumor region deformation or movement during radiotherapy. ${ }^{7,8}$ It combines a medical accelerator and modern imaging equipment and monitors tumor displacement through collecting patients' image information before or during treatment [such as cone beam computer tomography (CBCT), ${ }^{9,10}$ electronic portal imaging device (EPID), ${ }^{11,12}$ surface scanning, ${ }^{13-15}$ tumor marker of electromagnetic signal, ${ }^{16-18}$ and ultrasound-guided scanning ${ }^{19-21}$ ] in order to precisely irradiate the tumor region. However, the projection of rays into the patient's surface cannot be monitored by the above techniques. Moreover, the ionization chamber, film, and diode have been available for measuring projection of rays into the patient surface, but certain problems still exist in measured materials, such as no good tissue equivalence, low spatial resolution, invasive measurement, and no field matching measurement. The matching issue of adjacent radiation fields is still unsolved. It has become a hot and difficult issue about how to achieve monitoring matching of adjacent radiation fields during radiotherapy and reduce various side effects caused by mismatching.

Cherenkov emission is emitted when a charged particle traverses a dielectric medium with a velocity greater than the phase velocity of light in the medium. ${ }^{22}$ Cherenkov luminescence imaging (CLI) is an optical imaging modality to study charged particles of sufficient energy exceeding the Cherenkov emission light produced in biological tissue. ${ }^{23-26}$ With a typical continuous spectrum in biological tissue, the emitted light is then highly scattered and absorbed before reaching the surface, and the tissue optical properties tend to favor the transmission of the red-infrared light, where the Cherenkov emission is minimal. Due to the low light level, the detection of Cherenkov luminescence typical requires a charge-coupled device (CCD) coupled to a focusing optics lens, which is placed in a light-tight environment. Compared with traditional IGRT technique, CLI has many advantages, such as no radiation damage, fast imaging speed, high throughput, high spatial resolution, high sensitivity, low cost, and a wide range of applications, especially in monitoring the location of radiation rays in the body surface during radiotherapy. It can be used to effectively detect the matching information of adjacent radiation fields without extra radiation or invasive imaging. Therefore, CLI has a good application prospect in field matching in surface exposure regions during biological tissue radiotherapy. 
$\mathrm{Li}$ et al.: Using Cherenkov imaging to monitor the match line between photon...

In the context of radiotherapy, CLI has the unique capability of observing changes in the radiation fluence during treatment delivery and detecting the matching information of adjacent radiation fields. ${ }^{27,28}$ However, there are some unsolved issues for monitoring matching information of adjacent radiation fields with the CLI method. First, previous studies on field matching mainly discussed the accuracy of beam delivery without considering field mismatching projected in a surface exposure region due to introduced shifts. Second, previous works have been originally demonstrated in solid phantoms without considering the influence of biological tissue with an irregular surface and different optical properties. ${ }^{29-32}$ Third, matching has only been reported between photon and photons fields and electrons and electron fields without considering matching between photon and electron fields ${ }^{33}$ which often occurs in advanced breast cancer radiotherapy. Finally, previous works have not considered the effect of high dose rate photon fields' projection on the field matching. Therefore, the application of CLI in monitoring the matching of radiation fields during radiotherapy still needs to be studied. In this work, in view of the above unsolved issues, the matching of adjacent radiation fields was monitored quantitatively by CLI during irradiation of chicken tissue with yellow or black color. A model was introduced to quantify the matching error. The aim of this paper is to provide a biological experimental basis for monitoring matching of adjacent radiation fields due to introduced shifts during radiotherapy by the CLI technique.

\section{Methods}

\subsection{Radiation Delivery}

To explore the monitoring ability of CLI for the matching between photon and electron fields, all radiation treatment fields were delivered using a medical accelerator (Elekta VersaHD, Stockholm, Sweden) with CBCT. Cherenkov emission photons were captured using a camera with CCD during irradiation of chicken surfaces with yellow or black color. The generated photon energies included 6 and $10 \mathrm{MV}$ of conventional a dose rate of $600 \mathrm{MU} / \mathrm{min}$ and 6 and 10 flattening filter free (FFF) of high dose rate. The dose rate of 6 and $10 \mathrm{FFF}$ were 1200 and $2400 \mathrm{MU} / \mathrm{min}$, respectively. The generated electron energies included 4, 6, 8, 10, 12, and $15 \mathrm{MeV}$ with a dose rate of $600 \mathrm{MU} / \mathrm{min}$. The experiments were divided into two parts and three groups.

Part 1: the matching between photon and electron fields in the anterior/posterior (AP) direction in the surface of irradiated yellow chicken (group A) and black chicken (group B). In these experiments, the chicken was symmetrically placed on the accelerator treatment bed in supine position. The irradiation fields were divided into a lower photon irradiation field [Fig. 1(e)] and an upper electron irradiation field [Fig. 1(f)]. The photon field was delivered using collimator jaws set to $5 \times 5 \mathrm{~cm}^{2}$ at isocenter and $101.7 \mathrm{~cm}$ source-to-surface distance (SSD) [Fig. 1(e)]. the electron field was delivered using a $10 \times 10 \mathrm{~cm}^{2}$ cone with a $5 \times 5 \mathrm{~cm}^{2}$ field. The SSD for the electron field was set the same as the photon field to ensure that the machine head will not touch the chicken, as shown in Figs. 1(e) and 1(f). Photon-electron matched fields were delivered with known introduced shifts established using calculated table shifts. The following introduced shifts were tested: 10, 5, 2, and $0 \mathrm{~mm}$ toward inferior or superior direction using a couch motion.

Part 2: the matching between photon and electron fields in the surface of irradiated yellow chicken according to conventional breast radiotherapy plan (group C). In these experiments, the yellow chicken was symmetrically placed on the accelerator treatment bed in a supine position. The on-board imager integrated in the medical accelerator was adopted to acquire CBCT images before irradiation. The scan parameters for generating middle resolution images were as follows: $120 \mathrm{kV}, 300 \mathrm{mAs}$, S10, and F0. The scan angle was from $-178 \mathrm{deg}$ to $178 \mathrm{deg}$. The CBCT images were reconstructed with a thickness of $3 \mathrm{~mm}$ [Fig. 1(d)] and then transmitted to a Monaco V5.1 treatment planning system (Elekta, Stockholm, Sweden). To simulate the breast or chest wall irradiation and SCF or internal mammary lymph node irradiation for a breast cancer radiotherapy plan, the tangent photon fields of angles $330 \mathrm{deg}$ and $150 \mathrm{deg}$ were delivered to the chicken chest wall region, and a single electron field with an AP direction was delivered to the chicken neck region [Figs. 1(f)-1(h)]. The fields' isocenters were the same as the CBCT scanning center in order to ensure that an isocenter was adopted for all fields and the machine head 


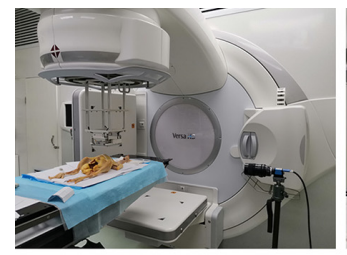

(a)

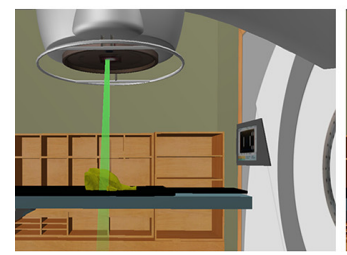

(e)

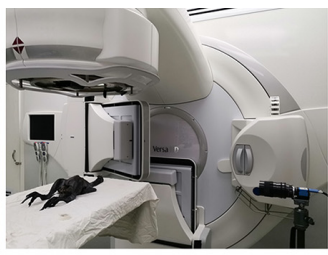

(b)

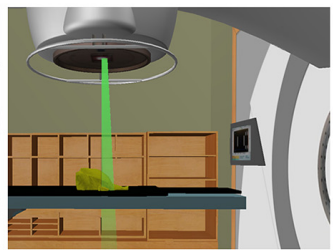

(f)

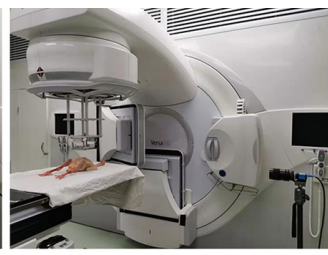

(c)

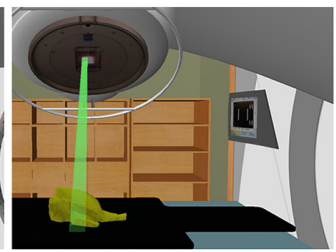

$(\mathrm{g})$

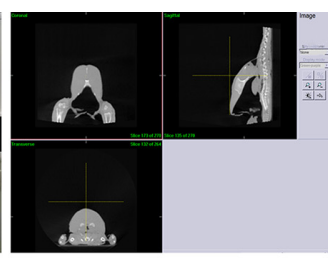

(d)

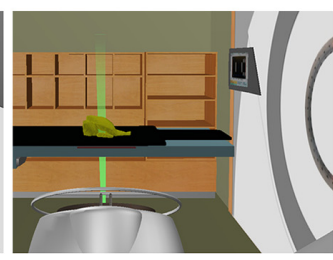

(h)

Fig. 1 The set up for monitoring matching between photon and electron fields. (a) Group A: yellow chicken experiment. (b) Group B: black chicken experiment. (c) Group C: yellow chicken experiment according to conventional breast radiotherapy plan. (d) CBCT image with three-direction plane. (e) Schematic illustration for photon field. (f) Schematic illustration for electron field. (g) Schematic illustration for photon field with gantry angle of 330 deg. (h) Schematic illustration for photon field with gantry angle of $150 \mathrm{deg}$.

would not touch the chicken [Figs. $1(\mathrm{~g})$ and 1(h)]. All photon fields were delivered using $5 \times$ $5 \mathrm{~cm}^{2}$ collimator jaws at the isocenter and all electron fields were delivered with $5 \times 5 \mathrm{~cm}^{2}$ field using a $10 \times 10 \mathrm{~cm}^{2}$ cone. After the plan was completely designed, it was transmitted to the Mosaiq network system and executed on the accelerator. Photon-electron matched fields were delivered with known introduced shifts established using calculated table shifts. Given that typical field junctions of $5 \mathrm{~mm}$ are employed clinically, ${ }^{34}$ the ability to verify field matching within $2 \mathrm{~mm}$ is ideal. Therefore, the following introduced shifts were tested: $10,5,2$, and $0 \mathrm{~mm}$ toward inferior or superior direction.

\subsection{Image Acquisition}

An industrial camera system (DMK 23U274) with a CCD was positioned to image the CLI signal in the chicken surface during irradiation. The camera was equipped with a fixed focal length and high aperture lens, which was fixed by a tripod [Fig. 1(a)]. In group A and group B, the surface of the lens was $80 \mathrm{~cm}$ away from the isocenter of the accelerator machine. In group $\mathrm{C}$, the surface of the lens was $130.5 \mathrm{~cm}$ away from the isocenter of the accelerator machine in order to avoid colliding with the accelerator head during CBCT scanning. To decrease the noise signal and avoid CLI signal interference from surrounding lights, light-blocking tape was used to shield all light-emitting devices in the treatment room, which included fluorescent lamps, safety lights, air conditioning indicator lights, an EPID plate sign lamp, an x-ray volumetric imager detection plate lamp, the control box lamp of a bed hand, a display lamp, and ultrasound-guided head lights on the ceiling

Before the beginning of the image acquisition, the ruler with a standard scale was imaged at the accelerator isocenter plane and the corresponding relationship between the scale and the photo pixel was be established for quantitative analysis about the matching error. In the process of image acquisition, the highest gain of $36 \mathrm{~dB}$ and minimum framerate of $5 \mathrm{f} / \mathrm{s}$ were selected to obtain a high signal-to-noise ratio during image acquisition. To optimize the integration acquisition time, the integration time was increased until the maximum signal intensity was observed. This verified that a minimum integration time of $1 \mathrm{~s}$ for photon irradiation and $1.2 \mathrm{~s}$ for electron irradiation were optimal to capture a maximum amount of Cherenkov signal. However, CLI signals could not be detected when the above integration time was adopted in group B. Next, the integration time was increased until relatively clear CLI signal images could be obtained. This verified that a minimum integration time of $2 \mathrm{~s}$ for photon irradiation and $2.5 \mathrm{~s}$ for electron irradiation were optimal to capture relatively clear CLI signal images in group B. 


\subsection{Matching Error Analysis}

All images were processed and evaluated using MATLAB ${ }^{\circledR}$ software packages (R2015b). The background images were acquired with the same conditions before the irradiation (radiation off) and subtracted out of the measured CLI image during the process of image analysis. To remove the sparkle noisy pixels caused by high-energy photons hitting the CCD directly, each image was generated by median filtering over a stack of three images from repetitive measurements and then smoothed by a median filter with a kernel size of 10 pixels $\times 10$ pixels. ${ }^{35}$ First, with no introduced shifts, the photon-excited CLI image was added to the electron-excited CLI image as a reference image, as shown in Fig. 2. Second, reference images were added to the photographic view taken at the same time [Figs. 3(b) and 3(e)]. According to the red and blue arrows as shown in Figs. 3(b) and 3(e), the location and shape of chicken wings and chest wall silhouettes in reference to Cherenkov image were consistent with that in photographic view images, which indicated the accuracy of the reference image (Fig. 3). By comparing the fused image and calculated dose image as shown in Figs. 3(b), 3(c), 3(e), and 3(f), CLI signals in the reference images showed similar locations and shapes with the chicken surface dose calculated by the treatment planning system (TPS) (Fig. 3). Finally, it could be seen that the location and shape of the CLI signal were consistent with that of the treatment region and surface dose.

Fields were delivered with known introduced shifts. Changes in the Cherenkov intensity represented field-matching values between photon and electron fields, which was evaluated through the generation of difference mismatching images from reference images. The photon-excited CLI image with tested introduced shifts was added to the electron-excited CLI image of no introduced shifts as a shifted photon CLI image. The electron-excited CLI image with tested introduced shifts was added to the photon-excited CLI image of no introduced shifts as a shifted electron CLI image. Then the reference image was subtracted from the shifted photon or electron CLI image to obtain the field matching error (Fig. 4). As shown in Fig. 5, in the matching error measurement for the photon field, a solid yellow rectangle region was placed on the location of the chicken wing in the subtracted image. The profile and the corresponding full width at half maximum (FWHM) of the chosen region along the $X$ axis direction could be calculated using MATLAB ${ }^{\circledR}$ software and indirectly represented the matching result due to lower chest wall movement during photon field delivery. In the matching error measurement for the electron field, the solid yellow rectangle region was placed on the location of the chicken chest wall in the subtracted image. The profile FWHM of the chosen region profile along the $X$ axis direction could be calculated and indirectly represented the matching result due to upper chest wall movement during electron field delivery. The FWHM was calculated manually as the pixel length width at half maximum intensity in grayscale profile. The maximum intensity and

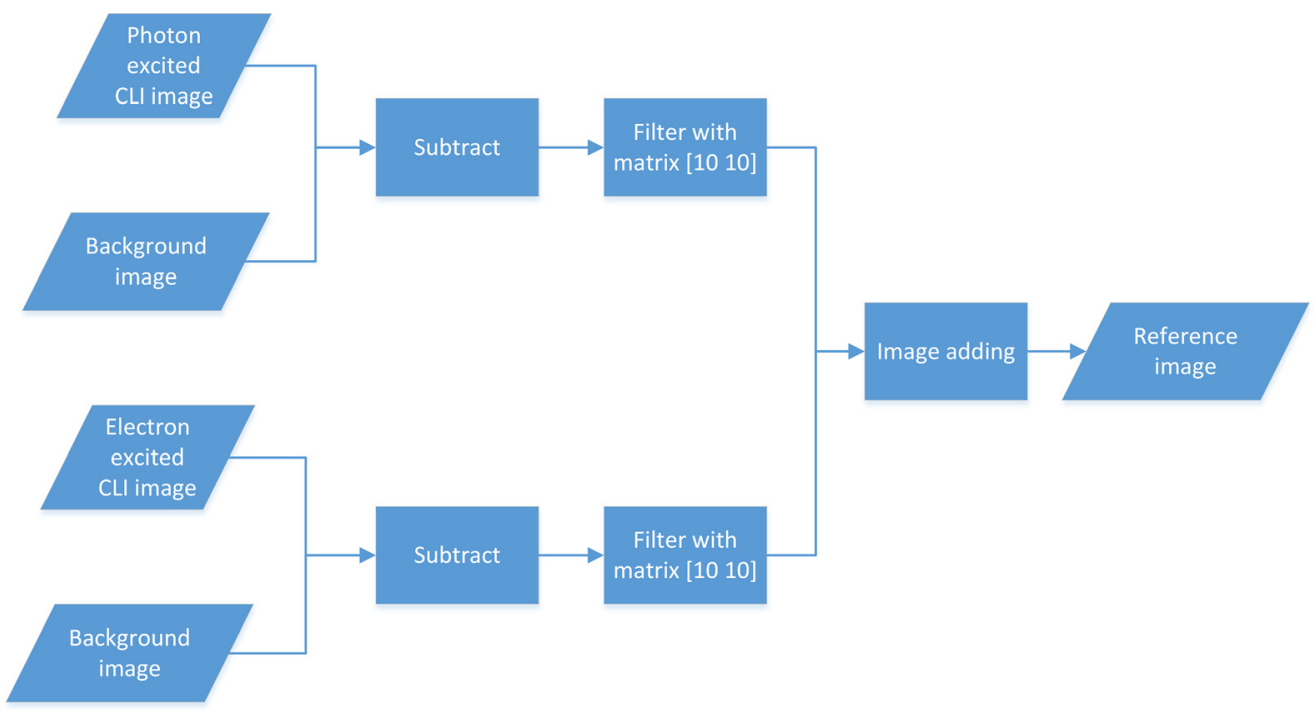

Fig. 2 With no introduced shifts, the photon-excited CLI image was added to the electron-excited CLI image of no introduced shifts as the reference image. 


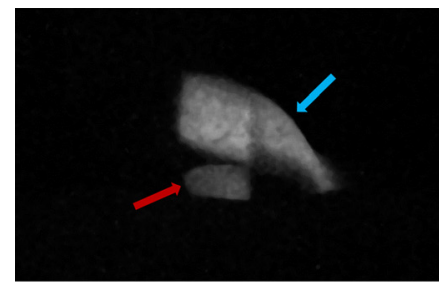

(a)

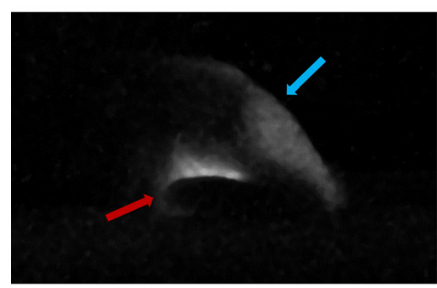

(d)

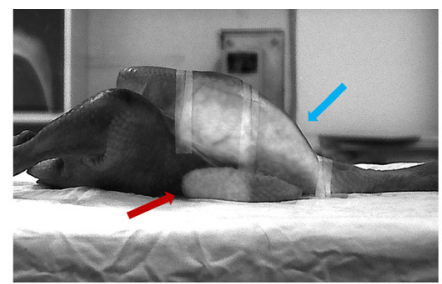

(b)

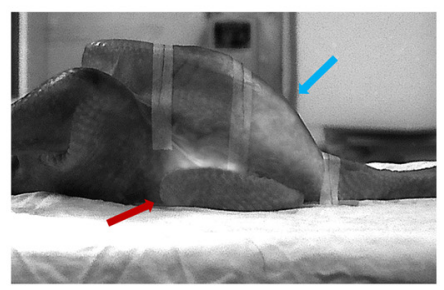

(e)

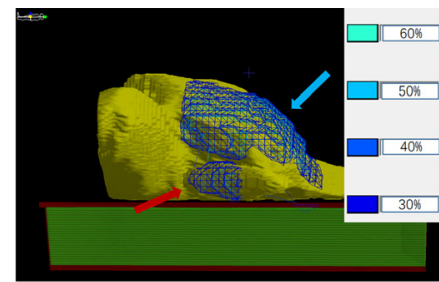

(c)

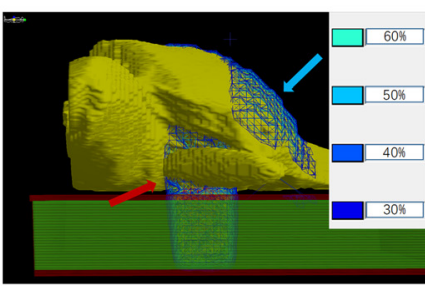

(f)

Fig. 3 Added and fused images in Group $C$ for photon fields with gantry angles of 330 deg and 150 deg. (a), (d) The reference CLI image between photon and electron fields (600 MU for photon field and $720 \mathrm{MU}$ for electron field). (b), (e) The fused image of reference CLI image and photograph. (c), (f) Calculated dose distribution in the chicken surface from TPS. Red or blue arrows pointed toward the position and shape of chicken wing or chest wall silhouettes in the CLI image, fused image, and calculated dose image, respectively.

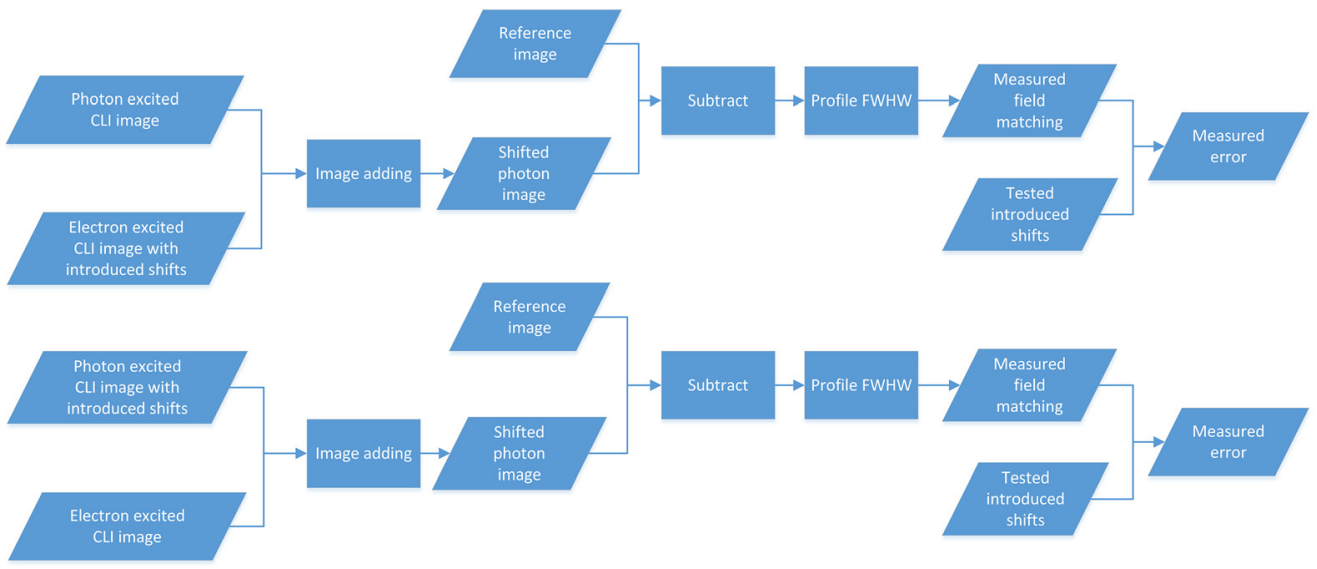

Fig. 4 Matching error measurement for photon and electron fields.

corresponding half maximum value were chosen manually using the MATLAB ${ }^{\circledR}$ data cursor tool in grayscale profile. With pixel-to-millimeter conversion factor, the FWHM was converted into millimeters as measured by the matching value. ${ }^{36}$ It was an easy and reliable method for calibrating distances in a clinical situation without the need for an additional device. In the results provided below, all matching values are absolute discrepancies in $\mathrm{mm}$.

\section{Results and Discussion}

\subsection{Result for Mean Grayscale Value of CLI Images}

Mean grayscale value (MGV) is defined as mean grayscale value of a chosen $5 \times 10 \mathrm{~cm}^{2}$ (with pixel-to-millimeter conversion) rectangle region for the photon field and a chosen $5 \times 7.5 \mathrm{~cm}^{2}$ (with pixel-to-millimeter conversion) rectangle region in the center of the CLI image. The chosen region was illustrated as a solid red rectangle region as shown in Figs. 7(a1), 7(e1), 7(i1), 7(m1), 


\section{Profile FWHW for photon field}

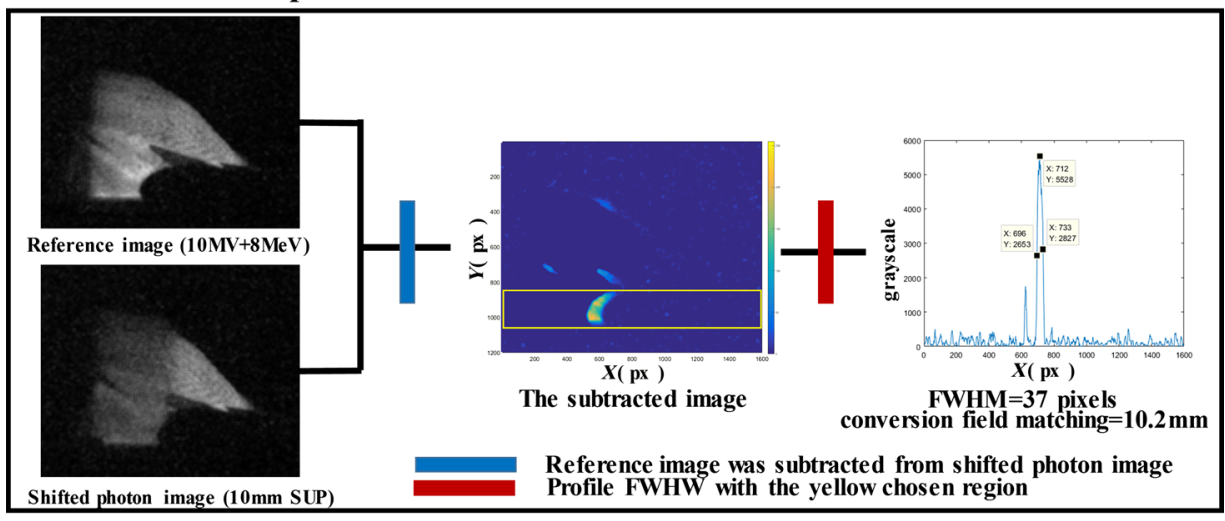

Profile FWHW for electron field

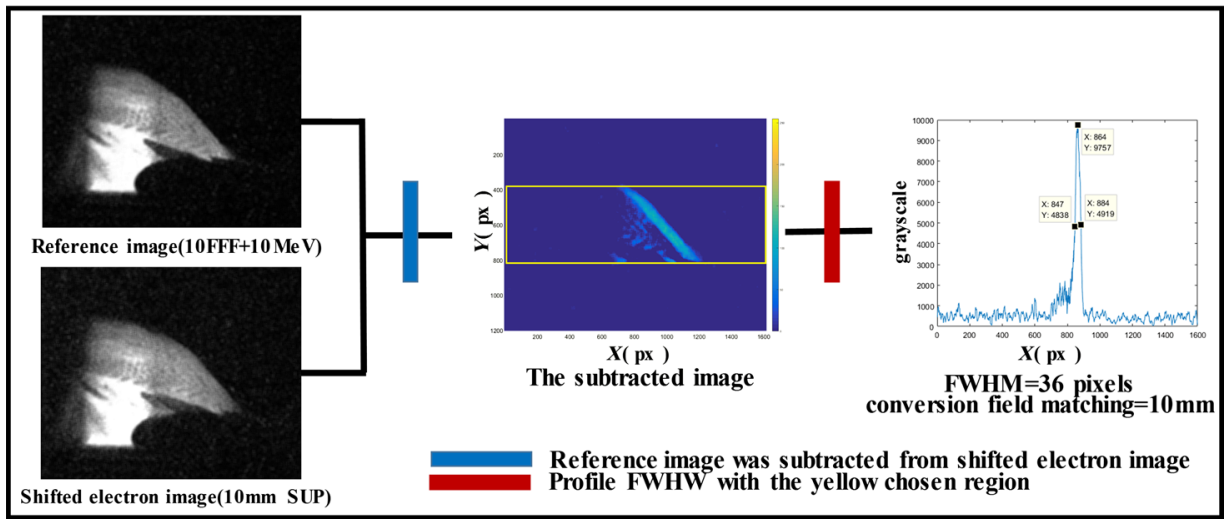

Fig. 5 Calculated process of profile FWHM and corresponding measured shifts due to introduced shifts during photon or electron field delivery.

and $8(\mathrm{a} 1), 8(\mathrm{~g} 1), 8(\mathrm{~m} 1)$. The MGV was calculated by averaging all the pixel values belonging to the chosen region using MATLAB ${ }^{\circledR}$ software. For electron fields, the MGV of images increased with the increase of energy (Fig. 6). According to the percent depth dose measured with parallel plate ionization chamber for the electron beam, the superficial dose is linear to the electron beam energies. The higher the energy, the higher the surface dose. Therefore, it was consistent with research by Zhang et al., ${ }^{30,37}$ which concluded that the Cherenkov signal intensity was directly proportional to the reference superficial dose data of corresponding electron energies simulated by the Monte Carlo method. For conversional dose rate photon fields, there was no obvious MGV difference with the increase of photon energy. However, the MGV for high dose rate fields was larger than that for conversional dose rate fields. This could be due to the high dose rate for 6 and 10 FFF fields, which were 2.33 to 3.66 times higher than that for conversional dose rate fields, which deposited larger superficial doses and excited larger Cherenkov photons per minute, resulting in larger MGV of the CLI image for high dose rate photon fields. Therefore, in the case of obtaining the same CLI intensity, integration time for high dose rate fields could be greatly reduced compared to that for conversional dose rate fields. Field matching efficiency for high dose rate fields could be greatly increased. The MGV results provide a reference for the selection of energy and efficiency assessment in field matching during radiotherapy.

In three experiments, the MGV of the CLI image for electron fields was greater than that for conversional dose rate photon fields, which was due to the deeper depth at max doses (roughly $1.5 \mathrm{~cm}$ for $6 \mathrm{MV}$ and $2.0 \mathrm{~cm}$ for $10 \mathrm{MV}$ ) for conversional dose rate photon fields, resulting in higher counts for electron fields. The MGV for photon fields with a gantry angle of $150 \mathrm{deg}$ was less than that for photon fields with a gantry angle of $330 \mathrm{deg}$. This could be caused by decreased Cherenkov photons collected from the exit side of the photon radiation. Different introduced shifts have great influence on the MGV for electron fields in the three experiments (Fig. 6). This was due to the variational contact areas including neck and upper chest wall between irradiation and the chicken surface, which were greatly affected by the different introduced shifts, 


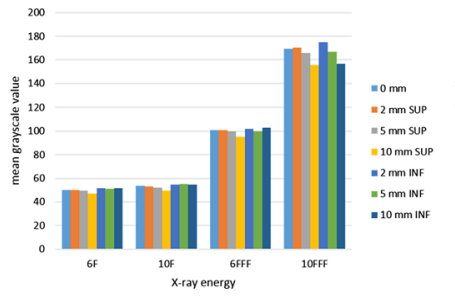

(a)

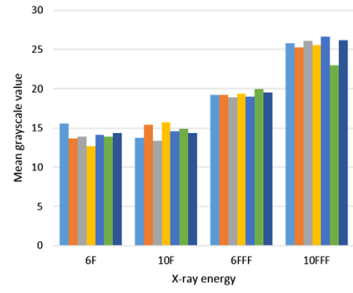

(c)

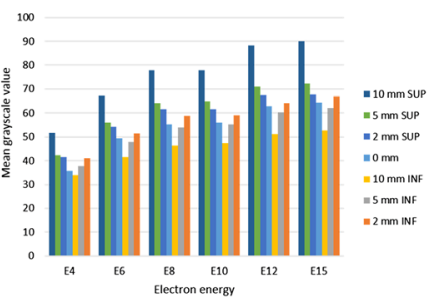

(b)

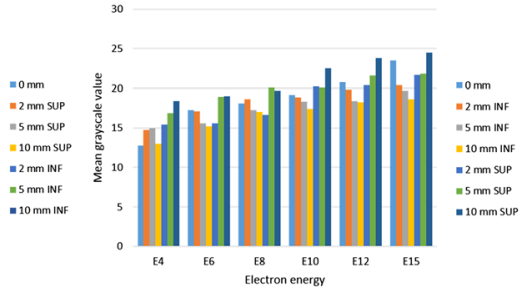

(d)

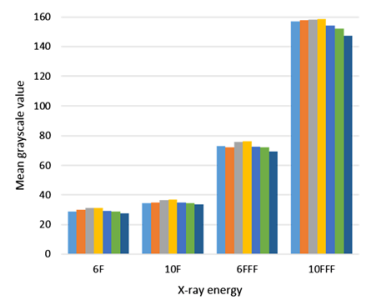

(e)

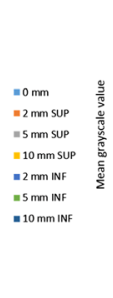

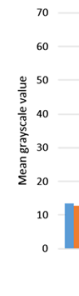

Fig. 6 MGV of photon and electron fields in the surface of irradiated yellow and black chicken (INF movement indicated chicken moved toward inferior direction. SUP movement indicated chicken moved toward superior direction.) (a) Photon field results in group A, (b) electron field results in group $A,(c)$ photon field results in group $B$, (d) electron field results in group $B,(e)$ photon field results with a gantry angle of $330 \mathrm{deg}$ in group $\mathrm{C}$, (f) photon field results with a gantry angle of 150 deg in group $\mathrm{C}, \mathrm{g}$ ) electron fields in group $\mathrm{C}$.
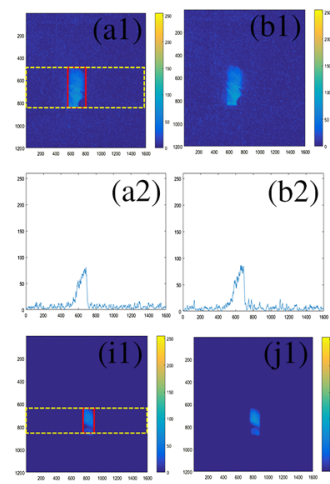

(i2)

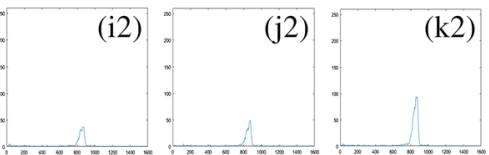

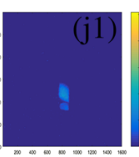

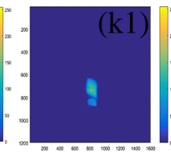

2)

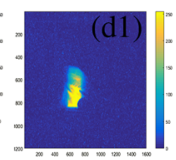

(c2)
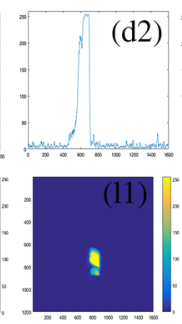

(12)

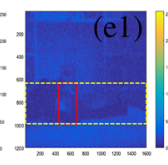

(e2)
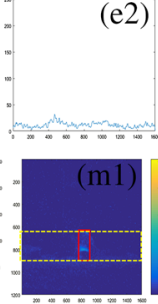

(m2)

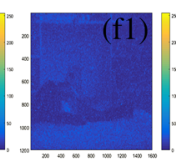

(f2)

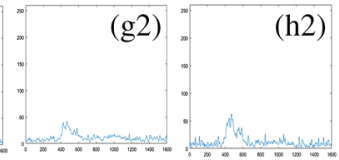

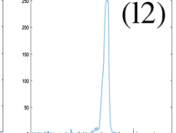
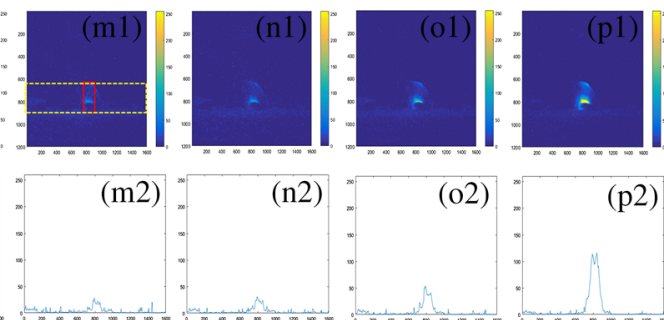

Fig. $7 \mathrm{CLI}$ images and corresponding grayscale profile with the yellow dotted region for photonexcited CLI images in group A [(a1), (a2) $6 \mathrm{MV}$; (b1), (b2) $10 \mathrm{MV}$, (c1), (c2) $6 \mathrm{FFF}$; and (d1), (d2) $10 \mathrm{FFF}$, group B [(e1), (e2) $6 \mathrm{MV}$; (f1), (f2) $10 \mathrm{MV}$; (g1), (g2) $6 \mathrm{FFF}$; and (h1), (h2) $10 \mathrm{FFF}]$, group $\mathrm{C}$ with a gantry angle of $330 \mathrm{deg}[(\mathrm{i} 1),(\mathrm{i} 2) 6 \mathrm{MV}$, (j1), (j2) $10 \mathrm{MV} ;(\mathrm{k} 1),(\mathrm{k} 2) 6 \mathrm{FFF}$; and (l1), (I2) $10 \mathrm{FFF}]$, and group $\mathrm{C}$ with a gantry angle of $150 \mathrm{deg}$ [(m1), (m2) $6 \mathrm{MV} ;(\mathrm{n} 1),(\mathrm{n} 2) 10 \mathrm{MV}$; (o1), (o2) 6 FFF; and (p1), (p2) 10 FFF]. 
Li et al.: Using Cherenkov imaging to monitor the match line between photon...

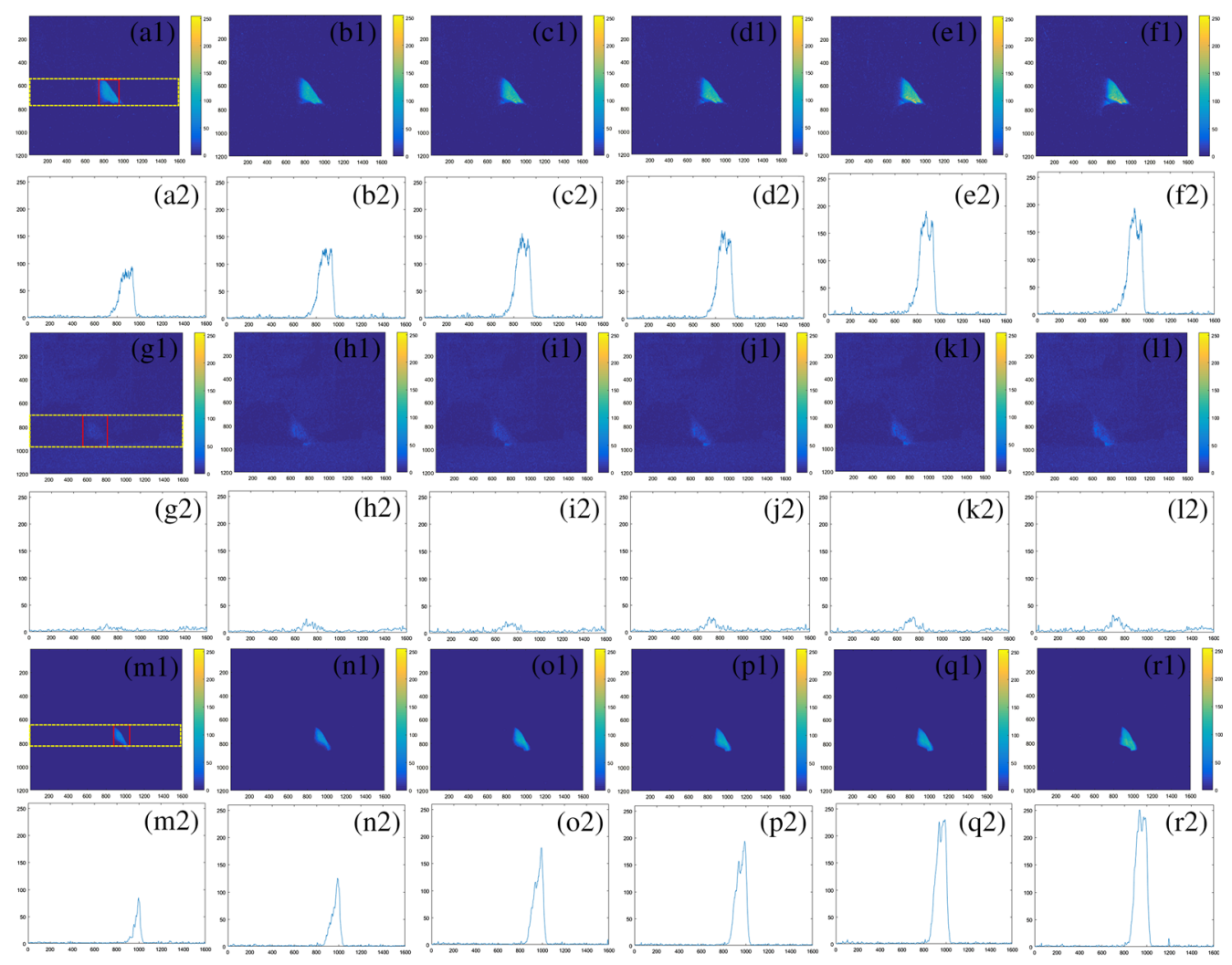

Fig. $8 \mathrm{CLI}$ images and corresponding grayscale profile with the yellow dotted region for electronexcited CLI images in group A [(a1), (a2) $4 \mathrm{MeV}$; (b1), (b2) $6 \mathrm{MeV}$; (c1), (c2) $8 \mathrm{MeV}$; (d1), (d2) $10 \mathrm{MeV}$; (e1), (e2) $12 \mathrm{MeV}$; and (f1), (f2) $15 \mathrm{MeV}]$, group B [(g1), (g2) $4 \mathrm{MeV}$; (h1), (h2) $6 \mathrm{MeV}$; (i1), (i2) $8 \mathrm{MeV}$; (j1), (j2) $10 \mathrm{MeV}$; (k1), (k2) $12 \mathrm{MeV;}$ and (l1), (I2) $15 \mathrm{MeV}]$, and group C [(m1), (m2) $4 \mathrm{MeV}$; (n1), (n2) $6 \mathrm{MeV}$; (o1), (o2) $8 \mathrm{MeV}$; (p1), (p2) $10 \mathrm{MeV}$; (q1), (q2) $12 \mathrm{MeV}$; and (r1), (r2) $15 \mathrm{MeV}]$.

resulting in a great variation in the number of Cherenkov photons under different introduced shifts for electron fields.

The grayscale profile value of CLI images (GPVCI) was defined as the averaged intensity profile value of a chosen rectangle region for photon fields and electron fields. The chosen region was illustrated as a dotted yellow rectangle region as shown in Figs. 7(a1), 7(e1), 7(i1), 7(m1) and $8(\mathrm{a} 1), 8(\mathrm{~g} 1), 8(\mathrm{~m} 1)$. The GPVCI for conventional dose rate photon fields was less than that for all electrons fields in group A (Fig. 7). This was consistent with the previous reports, which demonstrated that Cherenkov photons for photon fields were generated by secondary electrons through Compton scattering or photoelectric interaction and Cherenkov photons conversion efficiency for photon fields was lower than that for electron fields ${ }^{38}$ resulting in the above phenomenon. Compared with the results in group A, the MGV and GPVCI in group C were less for all photon and electron fields (Figs. 6-8). With the increase of CCD detection distance in group $\mathrm{C}$, the attenuation distance increased in the air for excited Cherenkov photons and the Cherenkov photons reaching the CCD detector decreased, resulting in decreases of MGV and GPVCI in group C. In addition, the GPVCI outside the chosen rectangle region in group C was significantly less than that in group A for electron fields and photon fields with a gantry angle of 330 deg which indicated that the noise may be attenuated greatly as the detected distance increased (Figs. 7 and 8).

\subsection{Result for Field Matching}

In group $\mathrm{A}$, the measured matching values were consistent with the tested introduced shifts values and all matching errors were within $1.5 \mathrm{~mm}$ (Fig. 9). Average discrepancies were 


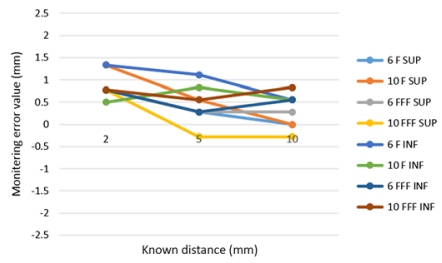

(a)

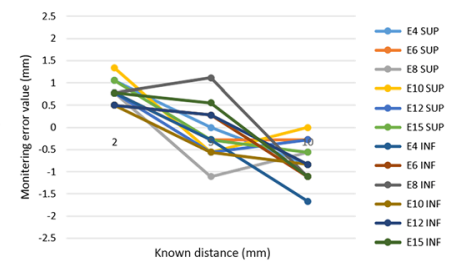

(b)

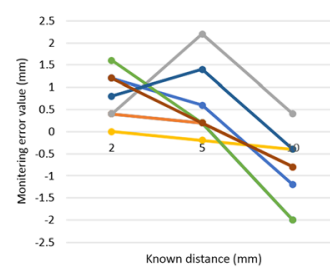

(c)
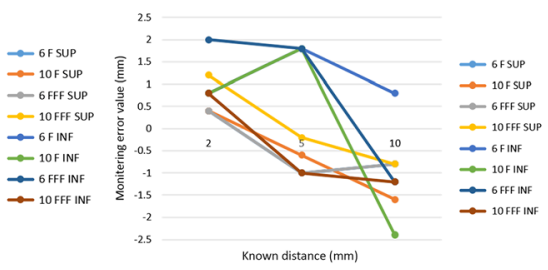

(d)

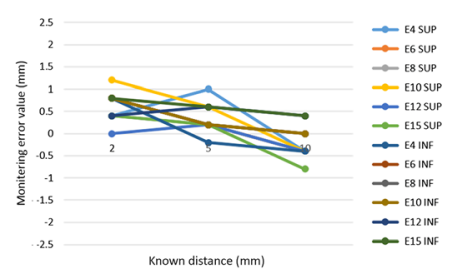

(e)

Fig. 9 Matching error values for photon fields and electron fields in surface of irradiated yellow chicken (INF movement indicated chicken moved toward inferior direction. SUP movement indicated chicken moved toward superior direction.) (a) Photon fields results in group A, (b) electron field results in group $A$, (c) photon field results with a gantry angle of 330 deg in group $C$, (d) photon field results with a gantry angle of $150 \mathrm{deg}$ in group $\mathrm{C}$, and (e) electron field results in group $\mathrm{C}$.

$0.59 \pm 0.35 \mathrm{~mm}$ for photon fields with $87 \%$ of measurements within $1 \mathrm{~mm}$. Average discrepancies were $0.68 \pm 0.37 \mathrm{~mm}$ for the electron field with $75 \%$ of measurements within $1 \mathrm{~mm}$. These errors appeared to have no energy dependence in group A. In group C, the measured matching values were consistent with the tested introduced shifts (Fig. 9). In the majority of experiments of group $\mathrm{C}$, the matching error values were within $2 \mathrm{~mm}$ for photon fields and $1.5 \mathrm{~mm}$ for electron fields. However, the matching error value was $2.2 \mathrm{~mm}$ for 6 FFF fields with $5 \mathrm{~mm}$ toward superior movement in the oblique incident field with a gantry angle of $330 \mathrm{deg}$. The matching error was $2.4 \mathrm{~mm}$ for the 10 -MV field with $10 \mathrm{~mm}$ toward inferior movement in the oblique incident field with a gantry angle of $150 \mathrm{deg}$. The average discrepancies were $0.80 \pm 0.65 \mathrm{~mm}$ and $1.07 \pm 0.57 \mathrm{~mm}$ for photon fields with gantry angles of $330 \mathrm{deg}$ and 150 deg with $66 \%$ and $65 \%$ of measurements within $1 \mathrm{~mm}$, respectively. The average discrepancies were $0.44 \pm 0.30 \mathrm{~mm}$ for electron fields with $94 \%$ of measurements within $1 \mathrm{~mm}$. These matching errors were not energy dependent in group $\mathrm{C}$.

Under AP irradiation in group A, the matching error values were within $1.5 \mathrm{~mm}$ for all photon fields and electron fields. Under oblique angles' irradiation in group C, all matching error values were within $2 \mathrm{~mm}$ for photon fields, with all but two being within $1.5 \mathrm{~mm}$ for electron fields. For electron fields, the matching error values in group $\mathrm{C}$ were more than that in group A (Fig. 9). The matching error values for AP photon fields were less than that for electron fields in group A. However, in group $\mathrm{C}$, the matching error values for oblique photon fields were more than that for electron fields. These results meant that the agreement between tested introduced shifts and measured values could be reduced with increasing field obliquity for photon fields. Earlier work by Zhang et al. ${ }^{39}$ has similar results, which demonstrated that this was due to the oblique field delivery, which extended the high-intensity field area in the direction of the field and broadened the field edge, effectively blurring the Cherenkov field edge and resulting in increased matching error values. These discrepancies, however, appeared to not be energy dependent. Interestingly, although the matching error values for electron fields were all within $1.5 \mathrm{~mm}$ in group A and group $\mathrm{C}$, the proportion less than $1 \mathrm{~mm}$ was significantly higher in group $\mathrm{C}$ with the same irradiation conditions. This could be caused by a decrease of the noise signal in the CLI image when the CCD detection distance increased in group C, which made the field boundary signal clearer and improved the detection accuracy. Additionally, it appeared to have downward trends with respect to larger introduced shifts in Fig. 9. The CLI intensity edge would be broadened due to signal noise and blurring CLI image, which mostly resulted in more matching value than introduced shifts in this study. It was most obvious for small introduced shifts. The matching discrepancies were consistently positive values for 2-mm introduced shifts in group A and group C. The impact of a broadening edge has been attenuated when introduced 
shifts decreased due to greater calculated cardinality, which resulted in downward trends with respect to larger introduced shifts.

It is important to note that for the AP irradiation photon field in group A, discrepancies were consistently positive values with average value of $0.55 \mathrm{~mm}$. This systematic discrepancy was observed for all introduced shifts and energies in group A, which indicated that a correction factor could be determined to decrease matching error values. The matching error could be less than $1 \mathrm{~mm}$ for 2-mm introduced shifts if the calculated error is subtracted from the systematic discrepancy for the AP photon field in group A. However, it was a limitation that a more percent error appeared for 2-mm introduced shifts by the proposed method. It may be caused by low image resolution $(36$ pixels $=10 \mathrm{~mm}$ ) with the $C C D$, which resulted in a larger pixel value of the profile FWHM and ultimately a larger percent error. Future works would focus on the highresolution camera for small introduced shifts and we are striving for a much smaller percent error. However, in the results of all oblique irradiation photon fields in group $\mathrm{C}$, there was no consistent discrepancy. This could be caused by the oblique field delivery, which extended the high-intensity field area in the direction of the field and broadened the field edge, resulting in a different deviation direction for matching error values.

\subsection{Result for High Dose Rate Photon Field}

In three experiments, the MGV and GPVCI for high dose rate fields were larger than that for conversional dose rate fields (Fig. 6). This could be due to the high dose rate for 6 and $10 \mathrm{FFF}$ fields at 2.33 to 3.66 times higher than that for conversional dose rate fields, which deposited larger superficial doses and excited larger Cherenkov photons, resulting in larger MGV of the CLI image for high dose rate photon fields. The higher the dose rate, the greater the MPV and GPVCI for high dose rate fields. In oblique irradiation photon fields, it was found that the variance and deviation distribution range of matching error values were less for high dose rate photon fields than that for conventional dose rate fields. These results could be explained by a larger deposited superficial dose and larger excited Cherenkov photons, which effectively increased the Cherenkov intensity and make the field edge clearer, resulting in matching values close to the tested introduced shifts for high dose rate photon fields.

Compared with the dose profile of conversional dose rate energies ( 6 and $10 \mathrm{~F}$ ) in water, a higher dose region appeared in the middle profile of the high dose rate energies ( 6 and $10 \mathrm{FFF}$ ). Details about the dose profile information are illustrated in Fig. S1 in the Supplementary Material. However, this did not happen in the GPVCI for photon fields, with no obvious distribution difference between high dose rate photon fields and conventional dose rate photon fields (Fig. 7). This could be due to small fields and irregular biological surface structures, which could weaken the signal of the high dose region for high dose rate photon fields and ultimately result in the above phenomenon.

\subsection{Effect of Color on Matching Result}

The Cherenkov effect produces a broad spectrum of light emission from UV down to near-infrared. In tissue, the emitted light is highly scattered and absorbed before leaving the surface and ultimately is collected by the CCD with a sensitive range in the visible wave band in this study. When the color of chicken tissue was black in group B, Cherenkov photons were highly absorbed by dark tissue with a high absorbed coefficient of the visible band, which resulted in dominance of Cherenkov emission in the spectrum of black tissue out of the sensitivity range of the CCD camera used and ultimately Cherenkov photons were hardly collected by the CCD with the same integration time as the other two groups. However, when the integration time was doubled, the number of unabsorbed photons increased and a CLI intensity image could be obtained with low intensity, large image noise, and fuzzy CLI intensity boundary. Previous research by Andreozzi et al. ${ }^{40}$ has similar results, which demonstrated that the darker tissue absorbed most Cherenkov photons, resulting in a Cherenkov signal being hardly collected by CCD. With the integration time increased in group B, the noise signal increased in CLI images, especially for photon fields (Figs. 7 and 8), which was similar to the one observed by Zhang et al. ${ }^{30}$ and Andreozzi et al. ${ }^{40}$ As a result, due to the large image noise and fuzzy 
CLI signal boundary in group B, the CLI signal could not be extracted from the surrounding environment. The matching between photon and electron fields could not be monitored with black biological tissue irradiation.

\section{Conclusions}

We have shown that CLI was acquired for matching monitoring between photon and electron fields due to introduced shifts in a biological tissue during radiotherapy. This capability is enabled by imaging the excited Cherenkov intensity in chicken surface with yellow or black color using a CCD. We concluded that the matching values between photon and electron fields were consistent with the tested introduced shifts for photon and electron fields. However, it is a limitation that the mismatching errors were introduced only by shifting the chicken tissue due to introduced shifts during radiotherapy. Further work will be required for customizing the plans for mismatching error research. For advanced breast cancer patients, photon fields and a single electron field have often been irradiated for breast and internal mammary lymph nodes, respectively. ${ }^{3,4,34}$ These adjacent fields need matching under complex geometrical regions. However, due to patients' respiratory movements or involuntary body movements during breast cancer radiotherapy, the mismatching between photon and electron fields could result in insufficient dosage or overdose in these regions. Therefore, the proposed technique may solve this problem and has the potential to apply to breast radiotherapy verification. Additionally, the proposed technique could be applied to craniospinal irradiation verification, which involves the matching of two whole brain fields and one to two spinal fields, depending on the anatomy of the patient. We believe, in the future, Cherenkov imaging could be incorporated into the treatment verification tools to monitor fields' matching during every fraction radiotherapy and further improve on treatment delivery and accuracy in clinical situations.

\section{Disclosures}

The authors declare no competing interests.

\section{Acknowledgments}

This work was supported by the financial support of the National Natural Science Foundation of China (Grant Nos. 61975232, 61775234, and 11604377), Qingdao National Laboratory for Marine Science and Technology of China (Grant No. QNLM2016ORP0111), the Major International (Regional) Joint Research Project of National Natural Science Foundation of China (Grant No. 42020104008), the Key Program of National Natural Science Foundation of China (Grant No. 41530422), and the National High Technology Research and Development Program of China (863 Program) (Grant No. 2012AA121101).

\section{Code, Data, and Materials Availability}

This study does not have associated code. Datasets generated during the present study were supported its results. All authors have declared the availability of data used in the research results reported in the paper and have provided specific access information or restrictions. No materials were used for the analysis in this study.

\section{References}

1. J. S. Vaidya et al., "Targeted radiotherapy for early breast cancer," Lancet 391(10115), 26-27 (2018).

2. J. R. Yarnold, "Selective avoidance of lymphatic irradiation in the conservative management of breast cancer," Radiother. Oncol. 2(2), 79-92 (1984). 
3. K. Long et al., "127: dosimetric comparison of 3D conformal radiation therapy (3DCRT) and volumetric ARC therapy (VMAT) in patients with bilateral breast cancer with indications for adjuvant radiation," Radiother. Oncol. 120, S48 (2016).

4. M. Roumeliotis et al., "Including internal mammary lymph nodes in radiation therapy for synchronous bilateral breast cancer: an international survey of treatment technique and clinical priorities," Breast Cancer Res. Treat. 171(2), 471-475 (2018).

5. L. A. Jarvis et al., "Cherenkov video imaging allows for the first visualization of radiation therapy in real time," Int. J. Radiat. Oncol. Biol. Phys. 89, 615-622 (2014).

6. W. Bogdanich, "The radiation boom: radiation offers new cures, and ways to do harm," N.Y. Times A1 (2010).

7. D. P. Gierga et al., "Quantification of respiration-induced abdominal tumor motion and its impact on IMRT dose distributions," Int. J. Radiat. Oncol. Biol. Phys. 58(5), 1584-1595 (2004).

8. S. Yoo et al., "Accuracy and efficiency of image-guided radiation therapy (IGRT) for preoperative partial breast radiosurgery," J. Radiosurg. SBRT 6(4), 295-301 (2020).

9. M. S. Ahlowalia et al., "Accuracy of CBCT for volumetric measurement of simulated periapical lesions," Int. Endod. J. 46(6), 538-546 (2012).

10. M. Cassetta et al., "How accurate is CBCT in measuring bone density? A comparative CBCT-CT in vitro study," Clin. Implant Dent. Relat. Res. 16(4), 471-478 (2014).

11. D. van Eeden, F. O'Reilly, and F. du Plessis, "Set-up error validation with EPID images: measurements vs Egs_cbct simulation," Rep. Pract. Oncol. Radiother. 24(6), 614-623 (2019).

12. E. S. Hsieh et al., "Can a commercially available EPID dosimetry system detect small daily patient setup errors for cranial IMRT/SRS," Pract. Radiat. Oncol. 7(4), e283-e290 (2017).

13. D. Reitz et al., "Real-time intra-fraction motion management in breast cancer radiotherapy: analysis of 2028 treatment sessions," Radiat. Oncol. 13(1), 128 (2018).

14. S. Schönecker et al., "Treatment planning and evaluation of gated radiotherapy in left-sided breast cancer patients using the CatalystTM/SentinelTM system for deep inspiration breathhold (DIBH)," Radiat. Oncol. 11(1), 143 (2016).

15. F. Crop et al., "Surface imaging, laser positioning or volumetric imaging for breast cancer with nodal involvement treated by helical TomoTherapy," J. Appl. Clin. Med. Phys. 17(5), 200-211 (2016).

16. T. R. Willoughby et al., "Target localization and real-time tracking using the Calypso 4D localization system in patients with localized prostate cancer," Int. J. Radiat. Oncol. Biol. Phys. 65(2), 528-534 (2006).

17. P. Kupelian et al., "Multi-institutional clinical experience with the Calypso system in localization and continuous, real-time monitoring of the prostate gland during external radiotherapy," Int. J. Radiat. Oncol. Biol. Phys. 67(4), 1088-1098 (2007).

18. K. R. Muralidhar et al., "Commissioning and quality assurance of Calypso four-dimensional target localization system in linear accelerator facility," J. Med. Phys. 38(3), 143-147 (2013).

19. T. O'Shea et al., "Review of ultrasound image guidance in external beam radiotherapy part II: intra-fraction motion management and novel applications," Phys. Med. Biol. 61(8), R90-R137 (2016).

20. U. Ricardi et al., "Three-dimensional ultrasound-based image-guided hypofractionated radiotherapy for intermediate-risk prostate cancer: results of a consecutive case series," Cancer Invest. 33(2), 23 (2014).

21. A. K. Richardson and P. Jacobs, "Intrafraction monitoring of prostate motion during radiotherapy using the Clarity? Autoscan transperineal ultrasound (TPUS) system," Radiography 23(4), 310-313 (2017).

22. P. A. Cerenkov, "Visible radiation produced by electrons moving in a medium with velocities exceeding that of light," Phys. Rev. 52(4), 378-379 (1937).

23. R. Robertson et al., "Optical imaging of Cerenkov light generation from positron-emitting radiotracers," Phys. Med. Biol. 54(16), N355-N365 (2009).

24. A. Ruggiero et al., "Cerenkov luminescence imaging of medical isotopes," J. Nucl. Med. 51(7), 1123-1130 (2010). 
25. A. E. Spinelli et al., "Cerenkov radiation allows in vivo optical imaging of positron emitting radiotracers," Phys. Med. Biol. 55(2), 483-495 (2010).

26. E. Ciarrocchi and N. Belcari, "Cerenkov luminescence imaging: physics principles and potential applications in biomedical sciences," EJNMMI Phys. 4(1), 14 (2017).

27. R. Zhang et al., "In vivo Cherenkov video imaging to verify whole breast irradiation treatment," Med. Phys. 41(6), 409-409 (2014).

28. R. Zhang et al., "Real-time in vivo Cherenkoscopy imaging during external beam radiation therapy," J. Biomed. Opt. 18, 110504 (2013).

29. A. K. Glaser et al., "Time-gated Cherenkov emission spectroscopy from linear accelerator irradiation of tissue phantoms," Opt. Lett. 37(7), 1193-1195 (2012).

30. R. Zhang et al., "Superficial dosimetry imaging of Čerenkov emission in electron beam radiotherapy of phantoms," Phys. Med. Biol. 58(16), 5477 (2013).

31. R. Zhang et al., "Čerenkov radiation emission and excited luminescence (CREL) sensitivity during external beam radiation therapy: Monte Carlo and tissue oxygenation phantom studies," Biomed. Opt. Express 3(10), 2381-2394 (2012).

32. R. W. Holt et al., "Cherenkov excited phosphorescence-based $\mathrm{pO}_{2}$ estimation during multi-beam radiation therapy: phantom and simulation studies," Phys. Med. Biol. 59(18), 5317-5328 (2014).

33. P. J. Black et al., "An investigation of clinical treatment field delivery verification using Cherenkov imaging: IMRT positioning shifts and field matching," Med. Phys. 46(1), 302-317 (2019).

34. E. A. Miles et al., "Dosimetry and field matching for radiotherapy to the breast and supraclavicular fossa," Radiother. Oncol. 91, 42-48 (2009).

35. L. Archambault, T. M. Briere, and S. Beddar, "Transient noise characterization and filtration in CCD cameras exposed to stray radiation from a medical linear accelerator," Med. Phys. 35(10), 4342-4351 (2008).

36. R. Zhang et al., "Beam and tissue factors affecting Cherenkov image intensity for quantitative entrance and exit dosimetry on human tissue," J. Biophotonics 10(5), 645-656 (2017).

37. R. Zhang et al., "Cherenkoscopy based patient positioning validation and movement tracking during post-lumpectomy whole breast radiation therapy," Phys. Med. Biol. 60(1), L1-L14 (2015).

38. A. K. Glaser et al., "A GAMOS plug-in for GEANT4 based Monte Carlo simulation of radiation-induced light transport in biological media," Biomed. Opt. Express 4(5), 741-759 (2013).

39. R. Zhang et al., "Superficial dosimetry imaging based on Čerenkov emission for external beam radiotherapy with megavoltage x-ray beam," Med. Phys. 40(10), 101914 (2013).

40. J. M. Andreozzi et al., "Camera selection for real-time in vivo radiation treatment verification systems using Cherenkov imaging," Med. Phys. 42(2), 994-1004 (2015).

Yi Li received his MS degree in biomedical engineering from Tsinghua University, Beijing, China, in 2012. Currently, he is pursuing his PhD in optics with the Xi' an Institute of Optics and Precision Mechanics, Chinese Academy of Sciences, Xi'an, China and in physics from Xi'an Jiaotong University, Xi'an, China. His research interests include nonlinear optics, Cherenkov imaging, and imaging processing.

Hongjun Liu received his $\mathrm{PhD}$ in optics from the Xi'an Institute of Optics and Precision Mechanics, Chinese Academy of Sciences, Xi'an, in 2002. Currently, he is a researcher with the State Key Laboratory of Transient Optics and Precision Mechanics, Xi' an Institute of Optics and Precision Mechanics. His current research interests include ultrafast optics, micronanooptics, and nonlinear optics.

Nan Huang received his MS and PhD degrees in optics from the Xi' an Institute of Optics and Precision Mechanics, Chinese Academy of Sciences, Xi'an, in 2009 and 2017, respectively. Currently, he is a researcher with the State Key Laboratory of Transient Optics and Precision Mechanics, Xi'an Institute of Optics and Precision Mechanics. His current research interests include optical wavelength conversion and sensitive detection of midinfrared light. 
Zhaolu Wang received his MS and PhD degrees in optics from the Xi' an Institute of Optics and Precision Mechanics, Chinese Academy of Sciences, Xi' an, in 2015. Currently, he is a researcher with the State Key Laboratory of Transient Optics and Precision Mechanics, Xi'an Institute of Optics and Precision Mechanics. His current research interests include weak-light signal enhancement and detection through nonlinear optics and all-optical signal processing through Silicon-based nonlinearity.

Chunmin Zhang received his $\mathrm{PhD}$ in optics from the Xi'an Institute of Optics and Precision Mechanics of CAS, Shaanxi, China in 2000. He is a professor, the director of Institute of Space Optics in Xi' an Jiaotong university. His research interests include imaging spectrum technology, optical remote sensing detection technology, atmospheric optics. He has published more than 200 research papers. 